\title{
APPLICATION OF SIMPLE CASCADE GPC WITH ROBUST BEHAVIOUR TO A SUGAR REFINERY
}

\author{
C. Bordons and E.F. Camacho \\ Dpto. Ingeniería de Sistemas y Automática, Univ. of Seville, Spain. Camino de los \\ Descubrimientos s/n, 41092 Sevilla, Spain. \\ Phone: +34-95-4487348Fax:+34-95-4487340E-mail:bordons, eduardo@cartuja.us.es
}

Keywords : Predictive Control, Process Control, Robust Control.

\begin{abstract}
This paper presents the application of a Generalized Predictive Controller (GPC) to sludge density control in a sugar factory. The loop is controlled by a cascade strategy, where both the master and the slave controllers are predictive ones. The control law is extremely simple to compute and the tuning is straightforward since a method to implement GPC previously developed by the authors which is very simple to implement and tune has been used. The controllers are embedded in the existing control system, needing the same computational requirements as PID routines. The original GPC algorithm is improved by the use of the so-called T polynomial, which increases the stability robustness by filtering the predictions in order to cope with model uncertainties and different process dynamics caused by changes in the process operating points.
\end{abstract}

\section{Introduction}

This paper shows an application of a GPC to a process in a sugar factory. The implementation was carried out by the authors in collaboration with the firm PROcISA. The sugar refinery is located in Peñafiel (Valladolid, Spain) and belongs to Ebro Agricolas. The control strategy runs in a ORSI Integral Cube Control System, where the GPC has been included as a library routine which can be incorporated in a control system as easily as the built-in PID routine.

Model Based Predictive Control (MBPC or MPC) is increasingly gaining acceptance and has been used in quite a number of applications across the world with spectacular results, first in the refinery and petrochemicals sector and nowadays also in many other fields in industry. There are many applications of predictive control successfully in use at the present time [10], not only in the process industry but also applications to the control of a diversity of processes [8], [11], [12]. MPC is particularly attrac- tive to staff with only a limited knowledge of control, because the concepts are very intuitive, and it can be used to control a great variety of processes, from those with relatively simple dynamics to other more complex ones. The term Model Predictive Control does not designate a specific control strategy but a very ample range of control methods which make an explicit use of a model of the process to obtain the control signal by minimizing a cost function.

The GPC method proposed by Clarke et al. [5] is a reasonable representative of this family of methods and has become one of the most popular MPC methods, being successfully implemented in many industrial applications [3]. As is well known, the basic idea of GPC is to calculate a sequence of future control signals in such a way that it minimizes a multistage cost function defined over a prediction horizon. The index to be optimized is the expectation of a quadratic function measuring the distance between the predicted system output and some predicted reference sequence over the horizon plus a quadratic function measuring the control effort.

A Generalized Predictive Controller results in a linear control law which is easy to implement once the controller parameters are known. The derivation of the GPC parameters requires, however, some mathematical complexities, which are difficult to solve in some industrial controllers. The industrial application of GPC in small control systems in industry has some difficulties that must be overcome. Apart from needing low computational requirements, it must be accepted by the plant operators. First, the tuning procedure must be simple enough, so that a GPC can be tuned as easily as a PID, and second, the controller must be robust, that is, it must behave well in the presence of the inevitable modelling errors.

The application shown here combines the power of predictive control with the simplicity and ease of use of the traditional controllers commonly found in industry. The simplicity is achieved by using a method developed by the authors which can be used with most processes in industry [1]. Due to this simplicity in the computational requirements, two GPC algorithms can be used as cascade controllers. In order to improve the robustness of the closed 
loop system, the $T$ polynomial has been added to the formulation.

The paper is organised as follows: Section 2 describes the application: the sludge density control in a sugar factory. The adaptation of the standard GPC algorithm to a wide class of industrial processes in order to reduce calculations and improve robustness is presented in section 3 ; section 4 is dedicated to the obtention of the plant model, section 5 shows some operating results and finally the conclusions of the work are presented in section 6

\section{Process Description}

The factory produces sugar from sugar-beet by means of a series of processes such as precipitation, cristalization, etc. The factory handles a large quantity of water; water is mainly necessary for producing steam in the boilers, for the diffusion process and for washing the sugar-beet. The water is not actually consumed so although used in various processes it is recoverable. The objective is that the factory should be completely self-sufficient with regard to water consumption.

One of the processes which consumes most water is washing the beet. Because it is a root plant growing below ground it always arrives at the factory covered in soil. Thus the first process necessary for the beet is to wash it. The raw material is washed using clean water to eliminate any remaining soil which logically must not enter into the sugar producing process. This operation results in large amounts of dirty water (sludge) being produced which must be recycled.

The recovery of clean water from this sludge is achieved by using the process shown in figure 1. A separating tank is used where sedimentation by gravity takes place, the water stays on the surface and the sludge sinks to the bottom. This separating tank is an open container with a diameter of 40 meters and an average depth of 5 meters. It is fitted with a blade which revolves at $0.05 \mathrm{rpm}$ and which ejects the thicker remains into a $35 \mathrm{~m}^{3}$ homogenizer cylinder from which it is pumped into the centrifuge which revolving at great speed separates the water from the soil by centrifugation. The water thus obtained returns to the separating tank from where the usable water is again drawn out.

The centrifuge works correctly for sludge flows of between 14 and $22 \mathrm{~m}^{3} / \mathrm{h}$ and a density which should be between 1100 and $1200 \mathrm{~g} / \mathrm{l}$. This density is the basic variable to be controlled as it must remain within this range to ensure the correct working of all the installation. This density depends upon the water recycled from the centrifuge to the separating tank and therefore on the sludge flow that circulates from the cylinder to the centrifuge.

It is difficult to model the relationship between the recirculation flow and the density, because various factors come into play and there is no clear relationship between said variables. However, it does seem clear that an increase

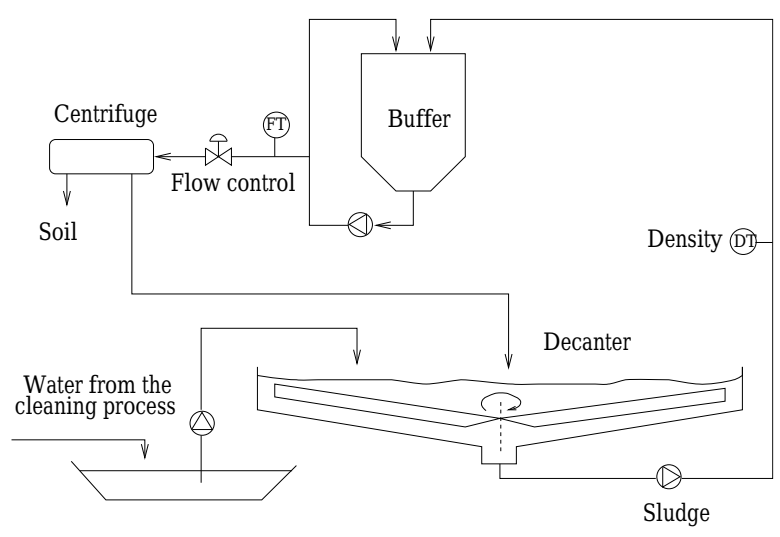

Figure 1: Water Recovery Process in a Sugar Refinery

in the flow causes more water to be recirculated and thus the density of the sludge to be decreased; a decrease of density has the opposite effect. An exhaustive modelling is rather complicated so a first order system with delay will be used to try to approximate the response.

An added problem is that the flow measurements are not very reliable due to the particles in suspension. Furthermore there are continuous blockages in the control valve which cause brusque alterations in the flow. On the other hand, because of the movement of the blades in the separating tank which stir the mud that will be pumped towards the centrifuge, the density measurements are not uniform because they keep falling like lumps of soil everytime the blade passes over the drain.

\section{Precomputed GPC}

This paper uses a formulation of Generalized Predictive Control (GPC) developed by the authors, easy to implement and tune, that is valid for the majority of industrial processes [1], [2]. The method makes use of the fact that a generalized predictive controller results in a control law that can be described with few parameters. The controller is valid for a wide class of processes in industry and a set of simple functions relating the controller parameters to the process parameters has been obtained. With this set of functions either a fixed or a selftuning GPC can be implemented in a straightforward manner.

Most processes in industry are high order systems that are not suitable for control purposes, but in general it is possible to approximate the behaviour of such high order processes with a simplified model consisting of a first order process combined with a dead time element [7]. This type of system is then described by the following transfer function:

$$
G(s)=\frac{K}{1+\tau s} e^{-s^{\tau} d}
$$

where $K$ is the process static gain, $\tau$ is the time constant or process lag, and $\tau_{d}$ is the dead time or delay. This 
model is widely used in industry to describe the dynamics of many processes, as shown by the popularity of the reaction curve method and the open loop Ziegler-Nichols PID tuning rules. Obviously better approximations could be obtained by using higher order models, but this would require identification packages which are not normally available in industry.

When the dead time $\tau_{d}$ is an integer multiple of the sampling time $T\left(\tau_{d}=d T\right)$, the corresponding discrete transfer function of equation (1) has the form:

$$
G\left(z^{-1}\right)=\frac{b z^{-1}}{1-a z^{-1}} z^{-d}
$$

where discrete parameters $a, b$ and $d$ can easily be derived from the continuous parameters by discretization of the continuous transfer function, resulting in the following expressions:

$$
a=e^{-\frac{T}{\tau}} \quad b=K(1-a) \quad d=\frac{\tau_{d}}{T}
$$

Therefore the CARIMA model used for the prediction is:

$$
A\left(z^{-1}\right) y(t)=B\left(z^{-1}\right) u(t-1)+\frac{C\left(z^{-1}\right)}{\triangle} \xi(t)
$$

where $C$ is the noise polynomial. If it is chosen equal to one, the model results:

$$
\left(1+a z^{-1}\right) y(t)=b z^{-1} u(t-d)+\frac{1}{\triangle} \xi(t)
$$

The predictions along the horizon from $t+d+1$ to $t+d+N$ can be calculated by means of the following equation:

$$
\begin{array}{r}
\hat{y}(t+d+j \mid t)=(1+a) \hat{y}(t+d+j-1 \mid t) \\
-a \hat{y}(t+d+j-2 \mid t)+b \triangle u(t+j-1)
\end{array}
$$

In [1] the GPC algorithm is derived for this kind of processes, leading to the control strategy shown in figure 2 . The plant parameters are used to compute the controller coefficients $\left(l_{y 1}, l_{y 2}, l_{r 1}\right)$ as described in [1]. These coefficients are precalculated as a function of the system pole $(a)$ and the control weighting factor $(\lambda)$ with horizons $N_{1}=d+1, N_{2}=d+N, N_{u}=N, N=15$. Notice that the dependence of these coefficients on parameter $b$ can be avoided if the controller is designed considering the plant to have a unitary static gain. The values $\hat{y}(t+d \mid t)$, $\hat{y}(t+d-1 \mid t)$ are obtained by the use of the prediction which basically consists of a model of the plant which is projected towards the future with the values of past inputs and outputs and only requires straightforward computation. The control signal is divided by the process static gain in order to get a system with a unitary static gain (since the controller coefficients have been calculated considering the system to have unitary gain). The control law is given by:

$$
\triangle u(t)=l_{y 1} \hat{y}(t+d \mid t)+l_{y 2} \hat{y}(t+d-1 \mid t)+l_{r 1} r(t)
$$

The control algorithm reduces to:

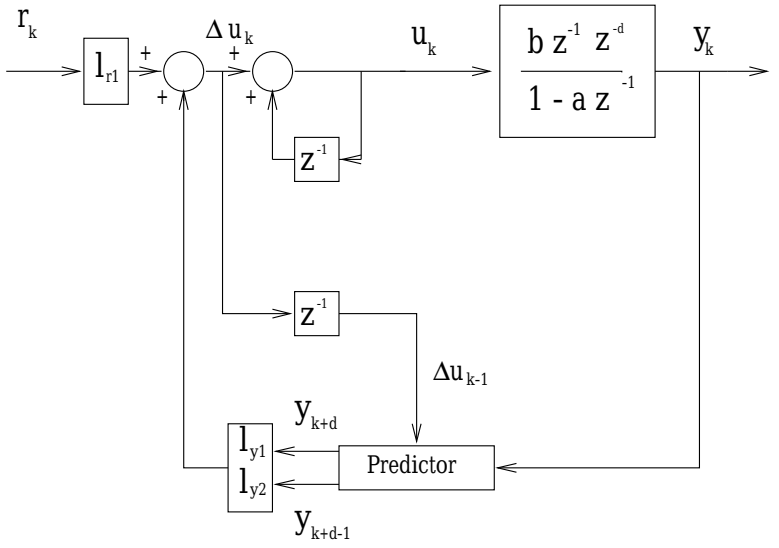

Figure 2: Control Scheme

1. Compute $k_{j i}$ as functions of the control weighting factor $\lambda$.

2. Make $l_{y i}=k_{1 i}+k_{2 i} \frac{\hat{a}}{k_{3 i}-\hat{a}}$ for $i=1,2$ and $l_{r 1}=-l_{y 1}-l_{y 2}$

3. Compute $\hat{y}(t+d \mid t)$ and $\hat{y}(t+d-1 \mid t)$ using equation (3) recursively.

4. Compute control signal $u(t)$ with: $\triangle u(t)=l_{y 1} \hat{y}(t+d \mid t)+l_{y 2} \hat{y}(t+d-1 \mid t)+l_{r 1} r(t)$

5. Divide the control signal by the static gain.

6. Go to step 2.

It can be seen that the algorithm is really simple and can be easily included in any commercial control system without complex calculation requirements. This algorithm has been successfully tested in some experimental plants. However, it has also been shown [2] that although it is rather robust to gain and time constant uncertainties, it has small robustness to dead time uncertainties, that are commonly found in real plants. That is why the algorithm must be modified to consider this circumstances, since the process to be controlled present that kind of uncertainty (as will be seen later).

The stability robustness of GPC can be improved with the use of an observer polynomial, the so-called $T\left(z^{-1}\right)$ polynomial. In [4] a reformulation of the standard GPC algorithm including this polynomial can be found. In order to do this, the CARIMA model is expressed in the form:

$$
A\left(z^{-1}\right) y(t)=B\left(z^{-1}\right) u(t-1)+\frac{T\left(z^{-1}\right)}{\triangle} \xi(t)
$$

Up to now the $T\left(z^{-1}\right)$ has been considered equal to 1 , describing the most common disturbances or as the colouring polynomial $C\left(z^{-1}\right)$. This polynomial is very difficult to obtain and in many cases it is considered as a design parameter. In consequence the predictions will not be optimal but higher robustness in the face of uncertainties can be achieved, in a similar interpretation as that used 


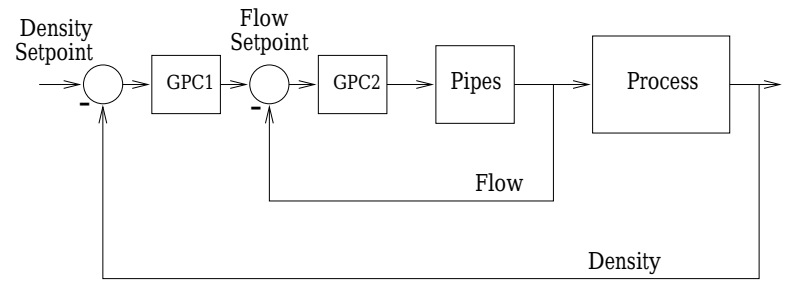

Figure 3: Cascade controllers

by Ljung [9]. This polynomial can be considered as a prefilter as well as an observer. The effective use of observers is known to play an essential role in the robust realization of predictive controllers (see [4] for the effect of prefiltering on robustness and [14] for guidelines for the selection of $T$ ).

The T polynomial can be easily added to the proposed formulation, computing the prediction with the values of inputs and outputs filtered by $T\left(z^{-1}\right)$. Then, the predictor works with $y^{f}(t)=\frac{y(t)}{T\left(z^{-1}\right)}$ and $u^{f}(t)=\frac{u(t)}{T\left(z^{-1}\right)}$. The actual prediction for the control law is computed as $\hat{y}(t+d)=$ $T\left(z^{-1}\right) \hat{y}^{f}(t+d)$.

The correct choice of the $T$ polynomial is a problem that has not completely been solved, although its effect on the robustness of the closed loop system has been analysed in several papers ([6], [4], [13], [15]). In this application, $T$ is made equal to $A\left(z^{-1}\right)\left(1-\beta z^{-1}\right)$, being $\beta$ a value close to the system pole, as suggested in [15].

\section{Model Attainment}

As has been explained, it is possible to control the density with the recirculation flow. As this flow suffers frequent disturbances due to the presence of discontinuities in the fluid composition, it is necessary to keep it controlled with another regulator. The control strategy is, therefore, going to be a cascade control, the density control acting on the remote setpoint of the local controller of the flow of the centrifuge (slave controller), see figure 3. This local control can be carried out using either a PI controller or a GPC indistinctively. The dynamics of this loop (a simple flow loop) does not justify itself the use of a GPC instead of a PI, but as the GPC routine is already installed in the control system and the computation time is similar in both cases, this loop was also used as a testbed for the controller. Besides, the tuning of the GPC is done automatically every time the system pole is updated.

A treatment of the density meter signal is needed to attain the model. Prior filtering is necessary to eliminate the noises and the effect of the blade strokes in the separating tank, in order to be able to work with an effective value indicative of the evolution of the actual density.

Following an initial analysis it is observed that the characteristic time of the system response is in the order of hours. In order to obtain a model, a step is provoked at the input, which is the flow setpoint, from 18 to $16 \mathrm{~m}^{3} / \mathrm{h}$. It is possible to obtain from the data a dead time of 2.6 hours, a time constant of 1 hour and a gain of $-25 \frac{\mathrm{g} / \mathrm{l}}{\mathrm{m}^{3} / \mathrm{h}}$.

\section{Operating Results}

The control was started up using filtering with the Tpolynomial. This polynomial is made equal to $A\left(z^{-1}\right)(1-$ $\beta z^{-1}$ ), with $\beta=0.9$. The control effort $\lambda$ was chosen equal to 0.1 . The local flow controller had previously been adjusted by the step response procedure obtaining $K=$ 2.5, $\tau=4 \mathrm{~s}$ and $\tau_{d}=10 \mathrm{~s}$ (notice the clear difference in the dynamics between the outer and inner loops).

For operative reasons it was necessary to limit the flow setpoint between 14 and $22 \mathrm{~m}^{3} / \mathrm{h}$, although it was not possible to use a predictive strategy with constraints because of computational limitations.

With the nominal model chosen for the density loop and with a sampling time of 12 minutes, the discrete parameters of the process model are given by:

$$
a=0.8187 \quad b=-0.4531 \quad d=13
$$

The controller coefficients can be computed (see [1]) calculating $k_{j i}(\lambda)$ and then $l_{y 1}, l_{y 2}$ and $l_{r 1}$ as functions of the system pole in the form:

$$
l_{y i}=k_{1 i}+k_{2 i} \frac{a}{k_{3 i}-a} \quad i=1,2 \quad l_{r}=-l_{y 1}-l_{y 2}
$$

$$
\begin{array}{ccc}
k_{11} & = & -\exp \left(0.3598-0.9127 \lambda+0.3165 \lambda^{2}\right) \\
k_{21}= & -\exp \left(0.0875-1.2309 \lambda+0.5086 \lambda^{2}\right) \\
k_{31}= & 1.05 \\
k_{12}= & \exp (-1.7383-0.40403 \lambda) \\
k_{22}= & \exp \left(-0.32157-0.81926 \lambda+0.3109 \lambda^{2}\right) \\
k_{32}= & 1.045
\end{array}
$$

As the system pole is in 0.8187 , if a value of $\lambda$ equal to 0.1 is chosen, the controller coefficients are

$$
l_{y 1}=-4.7454 \quad l_{y 2}=2.5930 \quad l_{r 1}=2.1524
$$

A similar procedure is used for the obtention of the fast flow controller, with values:

$$
l_{y 1}=-3.2448 \quad l_{y 2}=2.1294 \quad l_{r 1}=1.1154
$$

The control system operation is shown in the following diagrams. Note the time scale, where the slowness in the density evolution can be seen, two hours being necessary in order to carry out a change in the setpoint (see figure 4). Notice that the output shows an oscillatory signal added to the mean value. These oscillations are density changes due to the effect of the blades which stir the mud in the decanter, so the density measurements show this response because they keep falling like lumps of soil everytime the blade passes over the drain. 


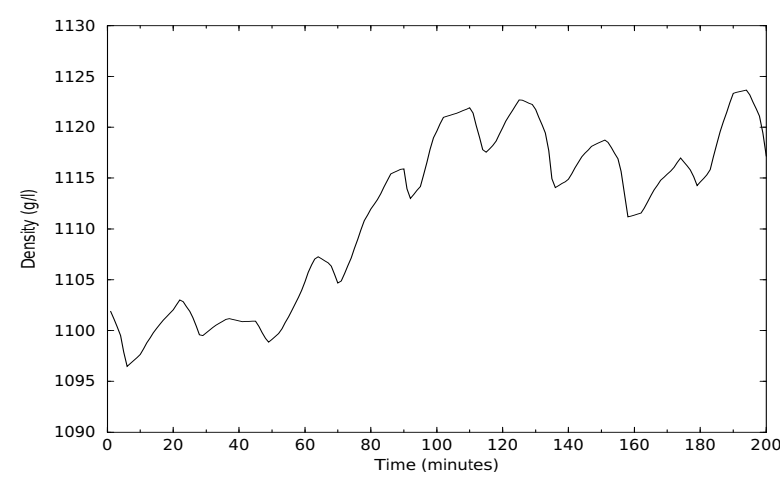

Figure 4: Setpoint change

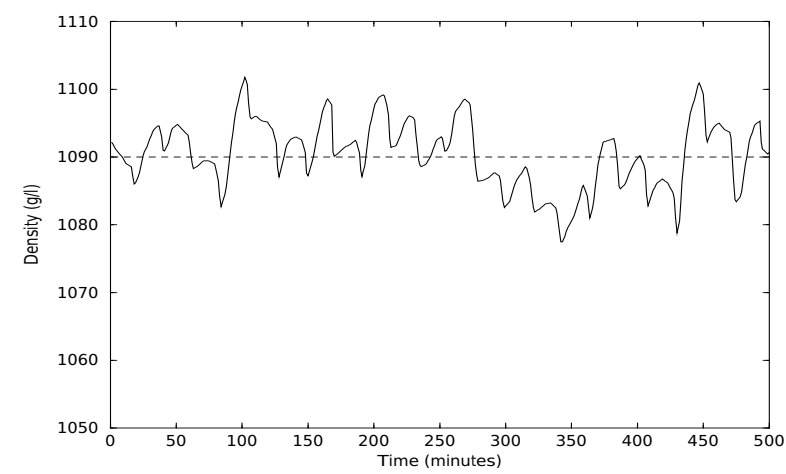

Figure 5: Disturbance rejection in the density loop

Note the importance of introducing the T-polynomial, given that the dead time obtained experimentally is not very precise and that also other tests showed that its value varied substantially from one situation to another. As is known, GPC is not very robust when faced with dead time modelling errors and the T-polynomial should be used in order to increase the robustness.

The control objective is to maintain the appropriate conditions for the centrifuge to work correctly. This requires to maintain the density at a constant value. The main density loop objective is to reject disturbances. Figure 5 show the behaviour of the controller during eight hours keeping the density at the right value of $1090 \mathrm{~g} / \mathrm{l}$.

The behaviour of the inner loop (the flow control loop) is drastically different from the density loop. The dynamics are faster, with rapid changes in the flow value. Figure 6 shows the behaviour of this loop when faced to setpoint changes provoked by the master controller. It can be seen that the signal is very noisy due to the difficulty of measuring a heterogeneous flow in a zone with large vibrations (due to the proximity to the centrifuge), but its means value follows the reference. Notice that the outer loop is open during this test in order to show the behaviour of the inner loop.

The controller interface allows the process parameters to be changed on line. The models are tuned by the oper-

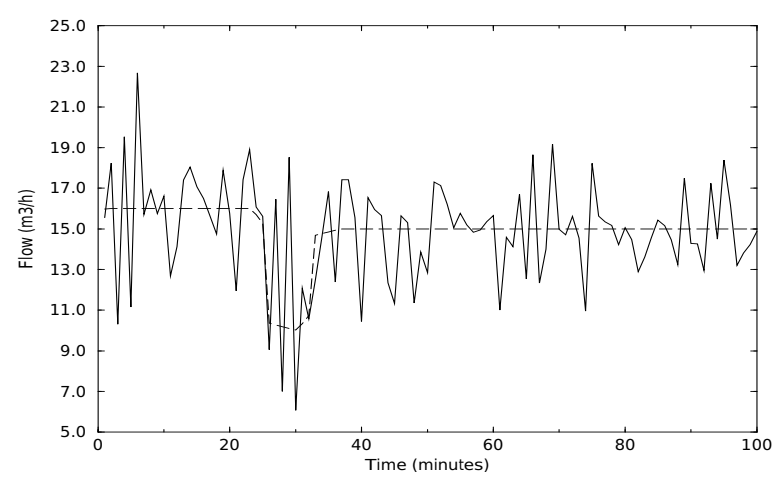

Figure 6: Setpoint following in the flow loop

ator as soon as a discrepancy between the actual and the predicted outputs (that appear on the screen) is detected.

It should be emphasised that this controller worked satisfactorily and without interruption until the end of the year's campaign, being handled without difficulty by the plant operators. The results obtained using this controller in terms of error variance improved the ones obtained in the last year's campaign, when the process was controlled by an operator, since they did not dare to tune a PID because of the long dead time.

\section{Conclusions}

An application of a Cascade Generalized Predictive Controller (GPC) to the sludge density control in a sugar factory has been presented. The control law was extremely simple to compute and the tuning was straightforward because a method to compute GPC previously developed by the authors which is very simple to implement and tune was used. The original GPC algorithm was improved by the use of the $\mathrm{T}$ polynomial to increases the stability robustness, since model uncertainties appeared when working at different operating points. The controller has been successfully working in the factory, showing a good behaviour.

The application shown here combines the power of predictive control with the simplicity and ease of use of the traditional controllers commonly found in industry, showing how a GPC can be easily used in clasical control structures like cascade control in the same way as PIDs.

\section{Acknowledgments}

The authors would like to acknowledge Juan Hermida from PROCISA by his interest and support in the development of the controller and the people from Ebro Agricolas by the facilities for testing the controller in the plant. The financial support of CICYT by projects TAP 96-884 y TAP 95-370 is gratefully appreciated. 


\section{References}

[1] C. Bordons and E.F. Camacho. Generalized Predictive Controller for a Wide Class of Industrial Process. IEEE Transaction on Control Systems Technology, $6(3): 372-387,1998$.

[2] E.F. Camacho and C. Bordons. Model Predictive Control in the Process Industry. Springer-Verlag, 1995.

[3] D. W. Clarke. Application of Generalized Predictive Control to Industrial Processes. IEEE Control Systems Magazine, 122:49-55, 1988.

[4] D.W. Clarke and C. Mohtadi. Properties of Generalized Predictive Control. Automatica, 25(6):859-875, 1989 .

[5] D.W. Clarke, C. Mohtadi, and P.S. Tuffs. Generalized Predictive Control - Part I. The Basic Algorithm. Automatica, 23(2):137-148, 1987.

[6] D.W. Clarke, C. Mohtadi, and P.S. Tuffs. Generalized Predictive Control - Part II. Extensions and Interpretations. Automatica, 23(2):149-160, 1987.

[7] P.B. Deshpande and R.H. Ash. Elements of Computer Process Control. ISA, 1981.

[8] D.A. Linkers and M. Mahfonf. Advances in ModelBased Predictive Control, chapter Generalized Predictive Control in Clinical Anaesthesia. Oxford University Press, 1994.

[9] L. Ljung. System Identification. Theory for the user. Prentice-Hall, 1987.

[10] S.J. Qin and T.A. Badgwell. "An Overview of Industrial Model Predictive Control Technology" in Chemical Process Control: Assessment and New Directions for Research. In AIChE Symposium Series 316, 93. Jeffrey C. Kantor, Carlos E. Garcia and Brice Carnahan Eds. 232-256, 1997.

[11] J. Richalet. Industrial Applications of Model Based Predictive Control. Automatica, 29(5):1251-1274, 1993.

[12] J. Richalet, A. Rault, J.L. Testud, and J. Papon. Model Predictive Heuristic Control: Application to Industrial Processes. Automatica, 14(2):413-428, 1978.

[13] B.D. Robinson and D.W. Clarke. Robustness effects of a prefilter in Generalized Predictive Control. Proceedings IEE, Part D, 138:2-8, 1991.

[14] T.W. Yoon and D.W. Clarke. Advances in ModelBased Predictive Control, chapter Towards Robust Adaptive Predictive Control, pages 402-414. Oxford University Press, 1994.
[15] T.W. Yoon and D.W. Clarke. Observer Design in Receding-Horizon Control. International Journal of Control, 2:151-171, 1995. 